to response. Mild adverse effects in 31\% included morning sedation and fatigue. (Prince JB, Wilens TE, Biederman J et al. Clonidine for sleep disturbances associated with attention-deficit hyperactivity disorder: a systematic chart review of 62 cases. I An Acad Child Adolesc Psychiatry May 1996;35:599-605). (Reprints: Dr Wilens, ACC 725, Massachusetts General Hospital, Boston, MA 02114).

COMMENT. Two thirds of the patients had medication-induced ADHDassociated sleep disturbance, mainly stimulants. The addition of clonidine in combination with stimulants such as methylphenidate in this study appeared to be safe and effective in correcting sleep disturbances. Fatalities have been reported using clonidine and methylphenidate together, and this combination therapy is being discouraged. The authors recommend further systematic assessment in large groups of children to clarify this issue. Clonidine is indicated in the treatment of $\mathrm{ADHD}$, tic disorders, and comorbid ADHD and tic disorders. Somnolence, the most common side-effect of clonidine, often reduces its usefulness.

Pharmacotherapy of ADHD is reviewed in a special article from the Massachusetts General Hospital. (Spencer T, Biederman J, Wilens T et al. I Am Acad Child Adolesc Psychiatry April 1996;35:409-432). The efficacy of stimulants in $70 \%$ of subjects has been documented in 155 controlled studies of 5,768 children, adolescents, and adults reported in the literature. Stimulants improve abnormal behaviors in ADHD, self-esteem, cognition, and social and family function. Response varied with age and comorbid conditions. The efficacy of tricyclic antidepressants in ADHD is also documented in more than 1000 subjects. Increased interest in comorbidity in ADHD has not been followed by related therapeutic advances. Data are limited on the response of medications in comorbid ADHD, and on the effects and safety of combined pharmacotherapy.

One of my readers has reminded me of studies carried out in the $1970 \mathrm{~s}$ regarding the effect of pyridoxine hydrochloride on hyperactive children having low levels of whole blood serotonin. Colleagues interested in revisiting the use of vitamin $\mathrm{B} 6$ in ADHD are referred to studies by Coleman $\mathrm{M}$, Coursin DB et al. Pediatrics 1975;55; Monogr neural Sci 1976;3:133-136; Biol Psychiatry 1979;14:741. Pyridoxine-induced sensory neuropathy has occurred with doses as low as $50 \mathrm{mg} /$ day when continued for months or years, and megadoses for the treatment of ADHD must be employed with caution. (see Ped Neur Briefs Feb 1995;9:14).

\title{
LEAD INTOXICATION IN CHILDREN WITH AUTISM
}

The incidence of reexposure to lead poisoning in 17 children with pervasive developmental disorders (PDD), including autism, compared to a randomly selected group of 30 children without PDD who were treated for plumbism over the same six year period, was evaluated by a retrospective chart review at the lead treatment program, Children's Hospital, Harvard Medical School, Massachusetts Poison Control System, Boston, MA. Despite close monitoring, inspection and lead hazard reduction or alternative housing, $75 \%$ of children with PDD were reexposed to lead compared to $23 \%$ without PDD. Those with PDD were older at diagnosis (46 vs 30 months) and had a longer period of elevated lead (39 vs 14 months) during management. (Shannon M, Graef JW. Lead intoxication in children with pervasive developmental disorders. Clin Toxicology March 1996;34:177-181). (Respond: Dr Michael Shannon, Children's Hospital, 300 Longwood Ave, Boston, MA 02115). 
COMMENT. Children with developmental delays and PDD are at increased risk of lead poisoning beyond 3 years of age. Children with PDD and other behavior disorders should be tested for lead at regular intervals beyond 4 years of age. More importantly, primary preventive measures designed to abate lead from the environment before lead intoxication occurs may be the only successful method of management.

\section{LEARNING DISABILITIES IN TOURETTE'S SYNDROME \& ADHD}

The psychosocial, psychoeducational, and neuropsychological data from 65 unmedicated, school-aged children with Tourette's syndrome (some with TS only and others with TS \& ADHD) and 27 comparison children were analysed at the Kennedy Krieger Institute and School of Medicine, Johns Hopkins University, Baltimore, MD. Leasrning disabilities were present in $23 \%$ of the total TS sample, but not in the TS-only group. The TS-only group had the highest mean FSIQ (117), exceeding the mean IQ of their parents. The TS+ADHD group had the lowest FSIQ. All TS groups had a poor performance on measures of choice reaction time, suggesting a deficit in preparedness to act or respond. The TS-only group was significantly weak in executive function or letter word fluency, but had age-appropriate scores on a timed neuromotor examination, and was better than TS+ADHD groups on untimed measures of visual-spatial skill. (Schuerholz LJ, Baumgardner TL, Singer HS, Reiss AL, Denckla MB. Neuropsychological status of children with Tourette's syndrome with and without attention deficit hyperactivity disorder. Neurology April 1996;46:958965). (Reprints: Dr Linda J Schuerholz, Kennedy Krieger Institute, Room 501, Developmental Cognitive Neurology, 707 N Broadway, Baltimore, MD 21205).

COMMENT. The authors suggest that in this sample of TS children, school difficulties are not synonymous with learning disability. Academic achievement could be related to timed linguistic efficiency, and cognitive slowing (so-called "bradyphrenia") may be out of proportion to cognitive impairment. A subtle "bradyphrenia" in patients with TS may account for some academic problems. Medications such as clonidine used to suppress tics may add to the cognitive slowing.

\section{DEPRENYL IN TOURETTE'S SYNDROME AND ADHD}

The efficacy of deprenyl, a monoamine oxidase inhibitor, was evaluated in the treatment of ADHD in 24 children and adolescents with comorbid Tourette's syndrome (TS) enrolled at the University of Rochester and Baylor College of Medicine. A double-blind placebo-controlled crossover design included two 8-week treatment periods separated by a 6-week washout period. A beneficial effect of deprenyl on tics was noted, but improvement of ADHD symptoms was limited to the first treatment period. (Feigin A et al. A controlled trial of deprenyl in children with Tourette's syndrome and attention deficit hyperactivity disorder. Neurology April 1996;46:965-968). (Reprints: Dr A Feigin, North Shore University Hospital, 444 Community Drive, Suite 206, Manhasset, NY 11030).

COMMENT. Deprenyl is metabolized to amphetamine and methamphetamine. By inhibiting breakdown of dopamine and increasing synaptic dopamine levels, deprenyl might be expected to worsen tics. These authors report a controlling effect on tics, possibly due to an influence on dopamine receptors. The failure to demonstrate a significant beneficial effect on ADHD could be explained by the crossover study design and patient dropout. 